\title{
Probing the dusty environment of the Seyfert 1 nucleus in NGC 3783 with MIDI/VLTI interferometry
}

\author{
T. Beckert, T. Driebe, S. F. Hönig, and G. Weigelt
}

\author{
Max-Planck-Institut für Radioastronomie, Auf dem Hügel 69, 53121 Bonn, Germany \\ e-mail: tbeckert@mpifr-bonn.mpg.de
}

Received 19 October 2007 / Accepted 30 May 2008

ABSTRACT

\begin{abstract}
Aims. We present mid-IR spectro-interferometry of the Seyfert type 1 nucleus of NGC 3783. The dusty circumnuclear environment is spatially resolved and the wavelength dependence of the compact emission is discussed.

Methods. The observations were carried out with the MIDI instrument at the Very Large Telescope Interferometer in the $N$-band. Spectra and visibilities were derived with a spectral resolution of $\lambda / \Delta \lambda \sim 30$ in the wavelength range from 8 to $13 \mu \mathrm{m}$. For the interpretation we developed a simple dusty disk model with a small and variable covering factor.

Results. At baselines of 65 and $69 \mathrm{~m}$, visibilities in the range of 0.4 to 0.7 were measured. The $N$-band spectra show a monotonic increase of the measured flux with wavelength with no apparent silicate feature around $10 \mu \mathrm{m}$. We find that the mid-IR emission from the nucleus can be reproduced by an extended dust disk or torus with a small covering factor of the radiating dust clouds.

Conclusions. Our mid-IR observations of NGC 3783 are consistent with a clumpy circumnuclear dust environment. The interpretation in terms of a dusty torus with a low covering factor supports a clumpy version of the unified scheme for AGN. The inferred sizes and luminosities are in good agreement with dust reverberation sizes and bolometric luminosities from optical and X-ray observations.
\end{abstract}

Key words. galaxies: active - galaxies: nuclei - galaxies: Seyfert - galaxies: individual: NGC 3783 - techniques: interferometric radiation mechanisms: thermal

\section{Introduction}

Unified scenarios of AGN suggest that the central continuum source is surrounded by a dusty molecular torus. This torus is thought to be responsible for aspect-angle-dependent obscuration of the central source (e.g., Antonucci 1993). Mid-infrared (MIR) interferometry of the Seyfert 2 nucleus in NGC 1068 (Jaffe et al. 2004; Poncelet et al. 2006) and the Circinus galaxy (Tristram et al. 2007) provided supporting evidence for the dusty torus model. The MIR emission of these nuclei was resolved by MIR interferometry with the MIDI instrument in both cases. The resolved structures are believed to be the IR-bright innermost region of the torus. In Seyfert type 1 sources the situation is not as clear, as NIR interferometry data on NGC 4151 obtained with the Keck interferometer (Swain et al. 2003) have been interpreted as accretion disk emission.

At a redshift of $z=0.0097$ (Theureau et al. 1998, $D=$ $44 \mathrm{Mpc}$ ), the galaxy NGC 3783 harbors a bright Seyfert 1 core with a black hole of $M_{\mathrm{BH}}=3.0 \pm 0.5 \times 10^{7} M_{\odot}$ as derived from reverberation mapping (Onken et al. 2004). NGC 3783 has been the target of extensive ultraviolet spectral monitoring (e.g., Reichert et al. 1994). A narrow iron K line is seen in XMM-Newton spectra (Reeves et al. 2004) consistent with an origin in Compton-thick matter such as a molecular and dusty torus. The optical spectrum shows blueward asymmetries in forbidden lines and broad lines of up to $3800 \mathrm{~km} \mathrm{~s}^{-1}$ in the broadest components (Evans 1988). The extended narrow line region of NGC 3783 seen with HST (Schmitt et al. 2003) appears halolike in [OIII] with a size of 1. ".9 (400 pc). The NIR spectrum of the nucleus (Reunanen et al. 2003) features a broad $\mathrm{Br} \gamma$ line and three coronal lines are detected. MIR spectra within a beam of 4".2 were presented by Roche et al. (1991) and showed an almost featureless spectrum with a $N$-band luminosity of $\sim 3 \times$ $10^{9} L_{\odot}$ in this aperture. VISIR photometry presented by Horst et al. (2006) show that NGC 3783 falls onto the MIR vs. hard $\mathrm{X}$-ray correlation for AGN with a rising flux of 0.66 to $0.72 \mathrm{Jy}$ across the $N$-band. The UV luminosity is estimated from $L_{\mathrm{UV}} \approx$ $6 \lambda L_{\lambda}(5100 \AA)$ to be $1-2 \times 10^{37} \mathrm{~W}$. This is consitent with the $L_{\text {bol }}-L_{X}$-relation (Marconi et al. 2004) which predicts a bolometric luminosity of $3 \times 10^{37} \mathrm{~W}$ for NGC 3783 . The accretion disk around the central black hole is therefore radiating at $\sim 10 \%$ of the Eddington limit. All these characteristics of a classical type 1 core suggest the presence of a dusty nuclear torus seen almost face-on. Direct evidence of this torus can only be obtained through high spatial resolution interferometry of the hot dust emission in the near- and mid-IR. In this paper we present the first spectro-interferometric observations of NGC 3783 with MIDI/VLTI. In Sect. 2 we describe the observations and data reduction. In Sect. 3 we develop a simple model for the circumnuclear dust distribution and discuss the implications for dusty tori in the unification scheme of AGN. Section 4 gives a short summary.

\section{Observations}

NGC 3783 was observed with MIDI on 27 and 30 May 2005 (Program 75.B-0697). A prism with a spectral resolution of $\lambda / \Delta \lambda=30$ at $10 \mu \mathrm{m}$ was used to obtain spectrally dispersed fringes between 8 and $13 \mu \mathrm{m}$. A detailed description of the observing procedure is given in Przygodda et al. (2003). We use the data reduction package EWS developed at Leiden Observatory 
Table 1. List of calibrators used in this work, together with $12 \mu \mathrm{m}$ fluxes $\left(F_{12}\right)$, and uniform-disk diameters $\left(d_{\mathrm{UD}}\right)$ of the MIDI observations.

\begin{tabular}{ccc}
\hline \hline Calibrator & $F_{12}(\mathrm{Jy})$ & $d_{\mathrm{UD}}(\mathrm{mas})$ \\
\hline HD 100407 & 11.6 & 2.41 \\
HD 116870 & 10.0 & 2.57 \\
HD 123139 & 54.4 & 5.25 \\
HD 169916 & 22.4 & 3.90 \\
HD 160668 & 28.8 & 2.30 \\
HD 152885 & 12.6 & 2.88 \\
HD 178345 & 10.6 & 2.50 \\
\hline
\end{tabular}

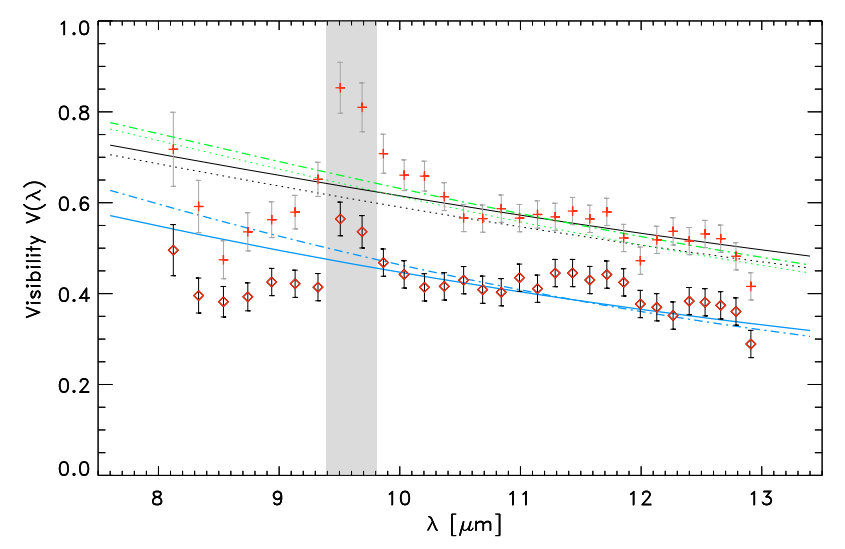

Fig. 1. Visibilities vs. wavelength of NGC 3783 in the $8-13 \mu \mathrm{m}$ range observed with MIDI on 30 May 2005. Visibilities from dataset 1 (diamonds) show lower visibilities than dataset 2 (crosses) for projected baselines of $B=68.6 \mathrm{~m}$ at $\mathrm{PA}=115^{\circ}$ and $B=64.9 \mathrm{~m}$ at $\mathrm{PA}=$ $120^{\circ}$. The gray area between $\lambda=9.4$ and $9.8 \mu \mathrm{m}$ is affected by the atmospheric ozone band, which could not be properly calibrated. The model visibilities A-1 (blue, dash-dotted) A-2 (green, dash-dotted), B-1 (blue, solid) and B-2 (black, solid) are shown for comparision. The models A-2 (green) and B-2 (black), modified for the longer baseline of $B=68.6 \mathrm{~m}$, are also plotted as dotted curves. This demostrates that the visibilty offset between Nos. 1 and 2 is not explained by the changing baseline when the visibility of each dataset is determined using the associated photometry shown in Fig. 2.

(combined MIA+EWS, ver.1.5.2) ${ }^{1}$. The EWS software first corrects for instrumental and atmospheric path differences before coherently adding the fringes (Jaffe 2004). We derive the interferometer transfer function at each wavelength (i.e. in each spectral channel) between 8 and $13 \mu \mathrm{m}$ by observing calibrators whose angular diameters are known (see Table 1).

In order to calibrate the visibility of NGC 3783 at a given wavelength, we use the mean of the transfer function values derived from all the calibrators observed on the same night as NGC 3783. The errors of the calibrated visibilities are estimated from errors on the raw visibilities and the standard deviation of the transfer function values at each wavelength (added in quadrature). The relative errors of the calibrated visibilities are typically $\sim 10 \%$. Figure 1 shows the calibrated visibilities of NGC 3783 from two datasets (Nos. 1 and 2 in the following) obtained close in time on 30 May 2005. The observed $N$-band visibilities are in the range of 0.4 to 0.7 . The wavelength dependence is weak within the errors from 8 to $13 \mu \mathrm{m}$ for both datasets. The projected baselines are $B=68.6 \mathrm{~m}$ and $64.9 \mathrm{~m}$ for dataset 1 and 2, respectively. We also include in Fig. 1 the visibilities of two different models described in Sect. 3.2 below. The

\footnotetext{
1 Available at http://www.mpia-hd.mpg.de/MIDISOFT/ and http://www . strw . leidenuniv .nl/nevec/MIDI/index . html
}

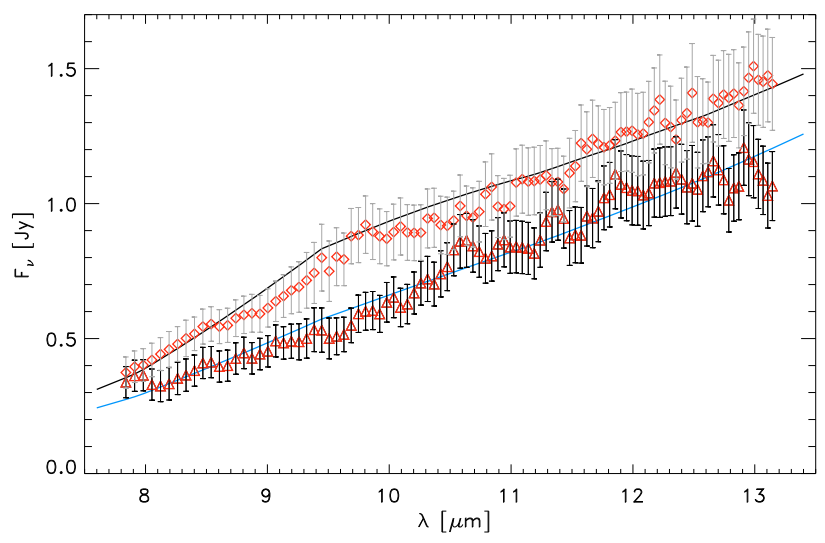

Fig. 2. MIDI $N$-band spectra of the nucleus of NGC 3783 in Jy vs. wavelength. The spectra have been calibrated and averaged against the reference stars of the night 30 May 2005. Dataset 1 and 2 are shown with diamonds (1) and triangles (2). The model spectra for B-1 (blue) and B-2 (black) of a clumpy and dusty torus corresponding to Fig. 1 and described in Sect. 3.2 are overplotted. The weak or absent silicate feature around $\lambda=9.7 \mu \mathrm{m}$ is a consequence of the large optical depth in the models.

wavelength region around $9.6 \mu \mathrm{m}$ (marked as a gray area in Figs. 1 and 3) is difficult to calibrate for faint targets with $F_{v} \approx 1$ Jy due to a strong atmospheric ozone band. This may explain the apparently greater visibilities in that wavelength region. In addition, we extracted absolute $N$-band spectra of NGC 3783 from MIDI single channel photometry. Interferometric calibrators observed on the same night as NGC 3783 and at similar airmasses serve as spectrophotometric standard stars to calibrate the spectra (see Table 1). The calibrated spectra of these stars are taken from Cohen et al. (1999). The two calibrated spectra of NGC 3783 are shown in Fig. 2. We estimate the errors of the spectra to be $10-20 \%$. The flux spectrum rises slowly with wavelength over the whole $8-13 \mu \mathrm{m}$ range. Neither the flux, in agreement with Roche et al. (1991), nor the visibility spectrum shows a prominent silicate feature at $\sim 10 \mu \mathrm{m}$.

Due to variable thermal emission from the sky and from inside the interferometer, the uncorrelated photometry measurements are subject to larger errors than the correlated flux measurement. In an alternative approach, we combined the spectra from both datasets to create the common total flux spectrum shown in Fig. 3. We used this to derive corrected visibilities for datasets 1 and 2 also included in Fig. 3. A comparision with model B-3 shows that the remaining difference between the two datasets can be attributed to the difference in the baseline.

\section{Discussion}

\subsection{General properties}

The first and most notable feature of the visibilities in Fig. 1 and the spectra in Fig. 2 is the absence of the $9.7 \mu \mathrm{m}$ absorption or emission feature of silicate dust expected to be present in the torus. This absence may be due to missing silicates in AGN dust, because of the lower sublimation temperature $T_{\mathrm{Si}} \sim 1000 \mathrm{~K}$ when compared with graphite grains $T_{\mathrm{C}} \sim 1500 \mathrm{~K}$. But the midIR emission is expected to come from $T \sim 300 \ldots 800 \mathrm{~K}$ dust, where both kinds of dust grains should be present. Alternatively, the dust grain size distribution maybe dominated by large grains, for which the silicate feature is less prominent. We have no direct evidence for this in NGC 3783, except for dust-reverberation arguments (see below). The third possibility, and our choice for 

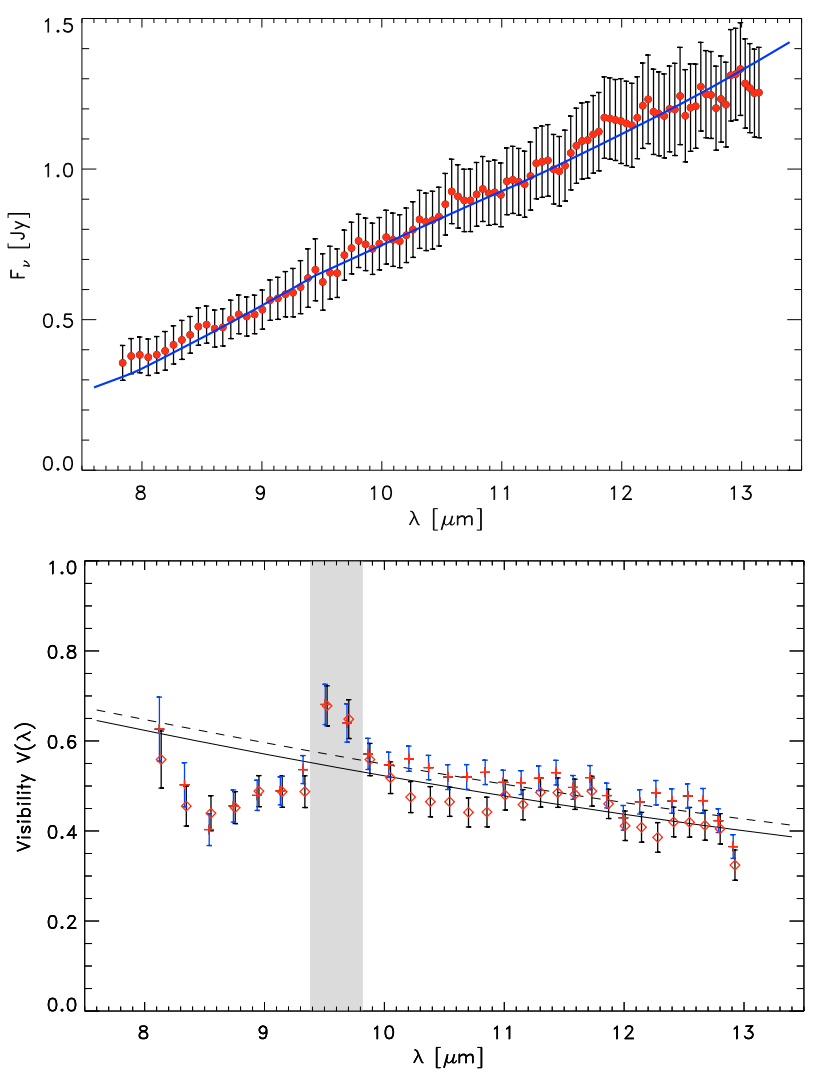

Fig. 3. Combined MIDI $N$-band spectrum (top) and visibilities (bottom) of NGC 3783. We used an arithmetic mean with errors for the two spectra from Fig. 2 and compare it with model B-3 (blue solid curve). The visibilities for datasets 1 (diamonds) and 2 (crosses) have been derived with the combined spectrum shown at the top. The visibilities for model B-3 for baseline $B=68.6 \mathrm{~m}$ (solid) and $64.9 \mathrm{~m}$ (dashed) are included. The measurements deviate substantially from the model in the 8.3 to $9.3 \mu \mathrm{m}$ region.

the models in Sect. 3.2 below, is a radiative transfer effect: for moderate optical depth in the $8-13 \mu$ m range with $\tau \sim 0.5$ and small temperature variations along the line of sight through the dusty torus, the emission spectrum approaches a black body and the feature is suppressed.

Secondly, the flux spectrum $F_{v}$ in Figs. 2 and 3 shows a linear rise with wavelength, in contrast to most radiative transfer simulations of face-on dusty AGN tori (e.g., Hönig et al. 2006). This may indicate the presence of additional, spatially distributed heating sources, which can increase the amount of dust at intermediate temperatures of 150 to $300 \mathrm{~K}$. Another possibility is a smaller covering factor of hot dust compared to cooler dust in a disk heated by the central engine. This idea is employed in the models below.

In addition, we find almost constant visibilities between $8-13 \mu \mathrm{m}$ suggesting a linear increase of the source size with wavelength. The derived visibilities are smaller than expected for a compact AGN dust torus, for which the NIR and MIR emission is dominated by a small region close to the dust sublimation radius. The diameter of the inner rim of the torus is expected to be 2.1 mas (see Eq. (2) below) corresponding to $V$ close to unity. A small dust covering factor in the models below can explain the data and implies a large extension of the MIR emission region.

Information on the torus inclination can be infered from spectropolarimetry in the optical 400 to $500 \mathrm{~nm}$ region, which shows a relatively large polarisation fraction of $\sim 1 \%$ at
$\mathrm{PA} \sim 130^{\circ}$ (Lira et al. 2007; Kishimoto, priv. comm.). The axis of the optical scattering region is therefore inclined with respect to our line of sight suggesting the minor axis of an elongated MIR disk to be close to the PA $\sim 120^{\circ}$ of our observations. The PA of the interferometric baselines for datasets 1 and 2 are very similar, so that we cannot constrain any non-circularity of the MIR emission from our data.

\subsection{Modeling visibilities and spectra with a dusty disk}

For the interpretation of the data presented above we employ a simplified version of the clumpy torus model (Beckert \& Duschl 2004; Hönig et al. 2006). A torus seen almost face-on $\left(i \approx 0^{\circ}\right)$ appears as a disk composed of dusty clouds. The inclination has almost no observable effect on spectra and colors as long as $i$ is smaller than the half opening angle of the torus. We assume single temperature clouds, where the cloud temperature depends only on the radius in the disk. For simplicity and comparison with other models we use a MRN (Mathis et al. 1977) grain size distribution between $0.005 \mu \mathrm{m}$ and $0.25 \mu \mathrm{m}$. The inner radius of the disk or torus is at $R_{\text {in }}$, where we assume a dust temperature of $T_{\text {in }}=1500 \mathrm{~K}$, which is suggested by NIR colors of type 1 AGN (Kishimoto et al. 2007). The local intensity $I_{v}(r)$ emitted by clouds is a modified black body $B(v, T)$ at temperature $T=$ $T_{\text {in }} \times\left(r / R_{\text {in }}\right)^{-\eta}$ with optical depth $\tau_{v}$, so that

$I_{v}(r)=B(v, T(r))\left(1-\mathrm{e}^{-\tau_{v}}\right)$.

We are interested in lines of sight through the disk parallel to the disk symmetry axis. For simplicity, we use a constant $\tau_{v}$ independent of the radius. The frequency dependence of $\tau_{v}$ is derived from the MRN distribution and a mixture of $50 \%$ silicates and $50 \%$ graphite grains with the optical properties taken from Weingartner \& Draine (2001, and references therein) ${ }^{2}$. For comparison we fix $\tau_{v}$ in the optical $\tau_{V}$ at $\lambda=560 \mathrm{~nm}$. For normal black body emission in radiative equilibrium with the central illuminating UV/optical radiation we expect an exponent $\eta=0.5$. This leads to an almost constant flux spectrum $F_{v}$ when integrated over the disk surface. We employ two modifications (models A and B) to fit the total flux spectrum in Fig. 2 (dash-dotted lines). Models A-1 and A-2 for datasets 1 and 2 assumes a different exponent $\eta=0.35$ (see Table 2). This is motivated by the equilibrium temperature of graphite grains (see e.g., Barvainis 1987; Kishimoto et al. 2007)

$\frac{T}{1500 \mathrm{~K}}=\left[\frac{L}{10^{37} \mathrm{~W}}\right]^{0.179}\left[\frac{R}{0.13 \mathrm{pc}}\right]^{-0.357}\left[\frac{\tilde{a}}{0.05 \mu \mathrm{m}}\right]^{-0.179}$

where the grain size $\tilde{a}=\left\langle a^{3}\right\rangle /\left\langle a^{2}\right\rangle$ is an average size for the grain size distribution. Such a model can explain the spectra in Fig. 2 but not the visibilities as shown in Fig. 1. We can accommodate the suggested large size of the dust emitting region by introducing a covering factor $f(r)$, for which we assume a radial dependence $f(r)=f_{0}\left(r / R_{\text {in }}\right)^{\xi}$. For model A, we use $\xi=0$ and $f_{0}$ can be adjusted to obtain the size of the source. The idea of a low covering factor is a direct consequence of clumpy torus models (see e.g., Hönig et al. 2006). In this scenario only clouds directly illuminated by the central source contribute significantly to the torus emission and due to the clumpy structure there is a non-zero probability for such clouds at all radii.

The implied AGN luminosity for models A-1 and A-2 are much smaller (see Table 2) than that derived from optical and

\footnotetext{
2 Files with tabulated optical constants have been taken from http:// www . astro.princeton . edu/ draine/dust/dust . diel . html
} 
Table 2. Dusty disk/torus model results. The covering factor $f_{0}$ and the exponents $\xi$ and $\eta$ for the covering factor and dust temperature are described in Sect. 3.2. $\tau_{V}$ is the optical depth along a vertical path through clouds in the disk taken at $560 \mathrm{~nm}$.

\begin{tabular}{ccccccc}
\hline \hline Model & $\tau_{V}$ & $f_{0}$ & $\xi$ & $\eta$ & $\begin{array}{c}R_{\text {in }} \\
{[\mathrm{pc}]}\end{array}$ & $\begin{array}{c}L_{\text {bol }} \\
{\left[10^{37} \mathrm{~W}\right]}\end{array}$ \\
\hline A-1 & 6 & 0.04 & 0 & 0.35 & 0.064 & 0.16 \\
A-2 & 12 & 0.06 & 0 & 0.35 & 0.04 & 0.065 \\
B-1 & 4 & $4.0 \times 10^{-3}$ & 0.8 & 0.5 & 0.30 & 3.7 \\
B-2 & 8 & $3.8 \times 10^{-3}$ & 0.9 & 0.5 & 0.21 & 1.7 \\
B-3 & 8 & $3.2 \times 10^{-3}$ & 0.9 & 0.5 & 0.23 & 2.2 \\
\hline
\end{tabular}

X-ray observations. We therefore concentrate in the following on models B-1 and B-2 which provide a better fit to the spectra in Fig. 2 and the visibilities in Fig. 1 with a running covering factor. All models exhibit decreasing visibilities with wavelength in Fig. 1 and both models A and B give acceptable fits to datasets 1 and 2 . The parameters of the models are summarized in Table 2. The inner torus radii of the models are determined by the total flux level and the covering factor in the combination $R_{\text {in }} / \sqrt{f_{0}}$. The AGN luminosity (assuming isotropic emission) in Table 2 is derived from Eq. (2). The higher luminosities for B-1, B-2 and B-3 are a direct consequence of the smaller covering factor at the inner boundary $R_{\text {in }}$. Note that the angular scale for $R_{\text {in }}=0.1 \mathrm{pc}$ is only 0.47 mas. The actual $T=300 \mathrm{~K}$ diameter is as large as 70 mas for model B-1 or 60 mas for A-1.

The visibilities derived with the combined total flux spectrum in Fig. 3 are much more consistent with each other. The difference between datasets 1 and 2 can be completely attributed to the difference in baseline length. This allows us to fit the long wavelength side the NGC 3783 datasets with a single model B-3 which is very similiar to B-2 (see Table 2). In the 8.3 to $9.3 \mu \mathrm{m}$ region the derived $V(\lambda)$ is substantially lower than suggested by all simple power-law models. NGC 3783 appears to be even larger than the models in this wavelength region.

\subsection{Implications for unified models}

Models of dusty nuclear tori suggested by the unified scheme of AGN (Antonucci 1993) require the dust to be contained in individual clouds or clumps (Krolik \& Begelman 1988). In recent years, models for the cloud size and cloud distribution (Beckert $\&$ Duschl 2004) and for the emission from a clumpy dust distribution (Nenkova et al. 2002; Hönig et al. 2006) have been developed. The dynamical model based on cloud collisions (Beckert \& Duschl 2004) suggests that a geometrically thick and obscuring torus is expected for high mass accretion rates close to the Eddington limit of the central black hole. From the X-ray and optical/UV luminosity of NGC 3783 an Eddington ratio of $10 \%$ has been inferred. Our simple model for the mid-IR emission suggests that the "torus" has a much smaller covering factor than expected for the collisional scenario.

Long term optical/IR photometry of NGC 3783 (Glass 2004, and references therein) suggests a time delay of $190 \mathrm{~d}$ between $U$ - and $L$-band ${ }^{3}$. This implies a physical path difference of $0.16 \mathrm{pc}$ or an angular scale of 0.75 mas, if the response is in the plane of the torus/disk. Given the uncertainties entering in Eq. (2) we find an agreement with the B-models within a factor of 2. For comparision, the NIR interferometry of NGC 4151 by Swain et al. (2003) suggests a more compact emission region

\footnotetext{
3 In an earlier paper (Glass 1992) a time delay of $85 \mathrm{~d}$ was suggested, which would imply a much smaller physical scale for the response.
}

such as an accretion disk or a ring at $R_{\text {in }}$. A discussion of dust sublimation radii, dust reverberation time delay and luminosity dependence can be found in Kishimoto et al. (2007). The inferred AGN luminosity from the B-models (Table 2) of $L_{\text {bol }} \approx 3 \times 10^{37} \mathrm{~W}$ or from the reverberation size via Eq. (2) are in close agreement with the $L_{\mathrm{bol}}-L_{\mathrm{X}}$-relation (Marconi et al. 2004).

\section{Summary}

We present MIDI/VLTI spectro-interferometry of the Seyfert 1 nucleus of NGC 3783 and spatially resolve the object between 8 and $13 \mu \mathrm{m}$. The observed visibilities suggest a significantly larger MIR emission region than the estimated dust sublimation size of 1.4 mas. Spectra and visibilities are noticeable for the apparent absence of a silicate feature around $10 \mu \mathrm{m}$. In addition the flux spectrum shows a linear increase with wavelength throughout the $N$-band. Consistent results are obtained with the use of an average total flux spectrum (Fig. 3) which shows that the incoherent flux measurements contribute the largest uncertainties. Our simple modeling simultaneously reproduces the observed visibilities and spectra by a dusty disk with a small covering factor seen face-on with a moderate optical depth. This model is consistent with recent clumpy torus models, although the covering factor of clouds in NGC 3783, which contribute to the MIR emission, is very small and rises outwards in the disk or torus.

Future near-IR and additional mid-IR interferometric measurements with different baseline length and covering a wider range of position angle are needed to confirm the present results and probe the validity of the simple models presented here.

Acknowledgements. T.B. thanks Christian Hummel and Sebastien Morel at ESO/Paranal for help and advice during the observations. We thank Makoto Kishimoto for stimulating discussions and helpful comments.

\section{References}

Antonucci, R. 1993, ARA\&A, 31, 473

Barvainis, R. 1987, ApJ, 320, 537

Beckert, T., \& Duschl, W. J. 2004, A\&A, 426, 445

Cohen, M., Walker, R. G., Carter, B., et al. 1999, AJ, 117, 1864

Evans, I. N. 1988, ApJS, 67, 373

Glass, I. S. 1992, MNRAS, 256, 23P

Glass, I. S. 2004, MNRAS, 350, 1049

Hönig, S. F., Beckert, T., Ohnaka, K., \& Weigelt, G. 2006, A\&A, 452, 459

Horst, H., Smette, A., Gandhi, P., \& Duschl, W. J. 2006, A\&A, 457, L17

Jaffe, W. 2004, SPIE Proc., 5491, 715

Jaffe, W., Meisenheimer, K., Röttgering, H. J. A., et al. 2004, Nature, 429, 47

Kishimoto, M., Hönig, S. F., Beckert, T., \& Weigelt, G. 2007, A\&A, 476, 713

Krolik, J. H., \& Begelman, M. C. 1988, ApJ, 329, 702

Lira, P., Kishimoto, M., Robinson, A., et al. 2007, in The Central Engine of Active Galactic Nuclei, ed. L. C. Ho, \& J.-W. Wang, ASP Conf. Ser., 373, 407

Marconi, A., Risaliti, G., Gilli, R., et al. 2004, MNRAS, 351, 169

Mathis, J. S., Rumpl, W., \& Nordsieck, K. H. 1977, ApJ, 217, 425

Nenkova, M., Ivezić, Ž., \& Elitzur, M. 2002, ApJ, 570, L9

Onken, C. A., Ferrarese, L., Merritt, D., et al. 2004, ApJ, 615, 645

Poncelet, A., Perrin, G., \& Sol, H. 2006, A\&A, 450, 483

Przygodda, F., Chesneau, O., Graser, U., Leinert, C., \& Morel, S. 2003, Ap\&SS, 286,85

Reeves, J. N., Nandra, K., George, I. M., et al. 2004, ApJ, 602, 648

Reichert, G. A., Rodriguez-Pascual, P. M., Alloin, D., et al. 1994, ApJ, 425, 582

Reunanen, J., Kotilainen, J. K., \& Prieto, M. A. 2003, MNRAS, 343, 192

Roche, P. F., Aitken, D. K., Smith, C. H., \& Ward, M. J. 1991, MNRAS, 248, 606

Schmitt, H. R., Donley, J. L., Antonucci, R. R. J., Hutchings, J. B., \& Kinney, A. L. 2003, ApJS, 148, 327

Swain, M., Vasisht, G., Akeson, R., et al. 2003, ApJ, 596, L163

Theureau, G., Bottinelli, L., Coudreau-Durand, N., et al. 1998, A\&AS, 130, 333

Tristram, K. R. W., Meisenheimer, K., Jaffe, W., et al. 2007, A\&A, 474, 837

Weingartner, J. C., \& Draine, B. T. 2001, ApJ, 548, 296 